\title{
A study of CME and type II shock kinematics based on coronal density measurement
}

\author{
K.-S. Cho ${ }^{1,2}$, J. Lee ${ }^{2}$, Y.-J. Moon ${ }^{1}$, M. Dryer ${ }^{3,4}$, S.-C. Bong ${ }^{1}$, Y.-H. Kim ${ }^{1}$, and Y. D. Park ${ }^{1}$ \\ ${ }^{1}$ Korea Astronomy and Space Science Institute, Hwaamdong, Yuseong-Gu, Daejeon, 305-348, Korea \\ e-mail: kscho@kasi.re.kr \\ 2 Center for Solar-Terrestrial Research, New Jersey Institute of Technology, Newark, NJ 07102, USA \\ 3 NOAA Space Environment Center, 325 Broadway, Boulder, CO 80303, USA \\ ${ }^{4}$ Exploration Physics International, Inc., Huntsville, AL 35806, USA
}

Received 27 January 2006 / Accepted 5 September 2006

ABSTRACT

\begin{abstract}
Aims. The aim of this paper is to determine location and speed of a coronal shock from a type II burst spectrum without relying on any coronal density model, and to use the result to discuss the relationship between the type II burst and Coronal Mass Ejection (CME). Methods. This study is made for the 2004 August 18 solar eruption observed by Green Bank Solar Radio Burst Spectrometer (GBSRBS) and a limb CME/streamer simultaneously detected by Mauna Loa Solar Observatory (MLSO) MK4 coronameter. We determine the background density distribution over the area of interest by inverting the MLSO MK4 polarization map taken just before the CME onset. Using the two-dimensional density distribution and the type II emission frequencies, we calculate the type II shock heights along several radial directions selected to encompass the entire position angles of the CME. We then compare these emission heights with those of the CME to determine at which position angle the type II burst propagated. Along the most plausible position angle, we finally determine the height and speed of the shock as functions of time.

Results. It turns out that the type II emission height calculated along a southern streamer best agrees to the observed height of the CME flank. Along this region, both the shock and CME moved at a speed ranging from 800 to $600 \mathrm{~km} \mathrm{~s}^{-1}$. We also found that the streamer boundary already had enhanced density compared to other parts before the CME and formed a low Alfvénic region.

Conclusions. We therefore conclude that the type II burst was generated at the interface of the CME flank and the streamer, as favorable for the shock formation.
\end{abstract}

Key words. shock waves - Sun: coronal mass ejections (CMEs) - Sun: radio radiation - Sun: corona - polarization methods: observational

\section{Introduction}

Type II radio bursts usually appear in a form of two frequency bands drifting with time, which correspond to the local plasma frequency and its harmonic, and are an important tracer for shock waves propagating away from the sun (Wild \& McCready 1950). From a typical density distribution we can infer that metric type II bursts are generated in the low corona, 1.2-2.5 $R_{\odot}$, and deca-hecto-kilometric type II bursts, in the interplanetary space. It is now agreed that decametric and longer wavelength type II bursts are due to Coronal Mass Ejections (CMEs), but the association of the metric type II bursts with CMEs remains as controversial (Gopalswamy et al. 1998; Cliver et al. 1999). Classen \& Aurass (2002) suggested that type II emission can be generated not only by CME shocks but also by the flare-produced shocks. In occasional radio imaging observations, type II sources usually appear broadly along the outer edge of the CMEs (e.g., Maia et al. 2000), but in some cases, type II bursts were found near the CME flank (e.g., Gary et al. 1984; Gopalswamy et al. 1997). It was also proposed that the association between metric type II bursts and CMEs depends on the local Alfvén speed as well as on CME energetics (Gopalswamy et al. 2001a, 2005).

In this paper, we present an analysis of an event that is designed to trace the type II burst from its generation in the low corona. Study of this single event would not entirely resolve the long-standing debate over the origin of all type II bursts.
We however believe that the present dataset and our new strategy have several advantages to offer an unusual insight into the origin of type II bursts. The advantages are as follows.

First, recent studies of CME dynamics have mostly used $\mathrm{SOHO} / \mathrm{LASCO} \mathrm{C} 2$ and C3 data, which provides excellent white light images in $2-32 R_{\odot}$. However type II shocks are believed to form in the lower corona, $1.2-2.5 R_{\odot}$, and one has to extrapolate the CME dynamics measured by LASCO observations back into the low corona typically assuming constant speed (e.g., Cho et al. 2003; Lara et al. 2003; Shanmugaraju et al. 2003). Such extrapolation is not desirable not only because it introduces additional uncertainty but because the bow shock surrounding a CME is not well represented by a single height at any given instant. We also note that CME leading edge is found at $2.2 R_{\odot}$ on average at the time of type II onset, which is just at the inner edge of the LASCO FOV (see Gopalswamy et al. 2005). To avoid these problems, we use the MK4 coronameter of Mauna Loa Solar Observatory (MLSO) to directly see the CME's kinematics in the type II formation region $\left(<3 R_{\odot}\right)$. The coronameter also has a high time cadence ( $3 \mathrm{~min}$ ) and thus is expected to provide us with near-simultaneous data of CME kinematics corresponding to the type II emission.

Second, most studies have used coronal density models in order to convert the frequencies of type II spectra to the height of the shock (Robinson \& Stewart 1985; Thompson et al. 1995). 
Use of a specific density model, however, leaves ambiguity, not because the density model is inaccurate, but because the real density distribution can vary with time and location (Bemporad et al. 2003; Guhathakurta \& Fisher 1998; Parenti et al. 2000). In the present study, we will estimate the coronal density using the polarization brightness data of MLSO MK4 coronameter to avoid the ambiguity introduced by using a fixed density model. A previous attempt to directly measure the density was made by Mancuso et al. (2003) who used SOHO Ultra Violet Coronagraph Spectrometer (UVCS) observations of the $\mathrm{O}$ VI doublet line to estimate type II shock speeds in the range from 1.5 to $2.3 R_{\odot}$.

Third, we argue that the coincidence of type II bursts with CMEs in terms of their onset times or speeds is insufficient evidence for identifying the origin of type II bursts. As an extreme case, we consider it possible that the flare, CME, and type II burst can occur almost simultaneously but at different places within the active region. As another, the CME may expand maintaining its shape so that the speed at the CME front may not be much different from the speed at the flank. In both cases we cannot locate the type II bursts with the timing or speed measurement alone. To resolve this ambiguity, the density distribution should be known, at least, in two-dimensional space so that we can distinguish between the flare site, CME front, and CME flank. In this regard, neither a density model-based result (usually given as a function of solar radius) nor a spectrometerbased result (usually one dimensional density distribution not in a radial direction) would help. Use of a two-dimensional polarization map is our advantage compared with other works.

In summary, we expect that the present dataset will allow to locate the type II burst source on the two dimensional plane without relying on the extrapolation of CME dynamics and density models, and that such result will help to resolve the ambiguity about the origin of the type II burst. Plan of this paper is as follows: in Sect. 2, we describe the radio and white-light observations. In Sect. 3, we present the result of the density measurement, location of the CME and the type II shock, and speed measurement. Finally, a conclusion is given in Sect. 4.

\section{Observations}

We study a CME event and the associated type II burst that occurred on 2004 August 18 mainly using the MLSO coronameter and Green Bank Solar Radio Burst Spectrometer (GBSRBS). The associated flare occurred at S14W90 and was classified as a GOES class X1.8 flare. The flare started at 17:30 UT and peaked at 17:40 UT with a duration time of $25 \mathrm{~min}$.

\subsection{White-light observation}

The MK4 coronameter produces white-light brightness and polarization maps every $3 \mathrm{~min}$ in the wavelength range from 700 to $950 \mathrm{~nm}$ with a field of view from 1.08 to $2.85 R_{\odot}$ (Elmore et al. 2003). We use the enhanced images available through the MLSO's website ${ }^{1}$ that are produced by applying an artificial vignetting function to the polarization brightness data. The angular and pixel resolutions of the MK4 coronameter are about 20" and 5.93", respectively.

Figure 1 shows the vignetted MK4 CME images taken at 17:44 UT (left panel) and 17:50 UT (right panel), respectively. We set two dotted guide lines, PA1 and PA2, to indicate the CME nose and flank, respectively, marking the heights of the

\footnotetext{
${ }^{1}$ http://mlso.hao.ucar.edu/cgi-bin/mlso_acoshome.cgi
}



Fig. 1. Vignetted MK4 negative images of the CME on 2004 August 18. The MK4 field of view is from 1.08 to $2.85 R_{\odot}$. Arrows indicate the CME heights measured along two dotted lines of the CME nose $\left(\mathrm{PA} 1=250^{\circ}\right)$ and $\mathrm{CME}$ flank $\left(\mathrm{PA} 2=230^{\circ}\right)$ near the helmet streamer, respectively.

CME front along each position angle with arrows. It is obvious to our eyes that the CME nose moved out along the position angle PA1. The height of the nose was about $2.0 R_{\odot}$ at 17:44 UT and $\sim 2.3 R_{\odot}$ at 17:50 UT. It was a little bit subtle to locate the CME flank at PA2, because the half width of the whole CME is about $30^{\circ}$ and PA2 is only $20^{\circ}$ away from PA1. It is however clear from a movie ${ }^{1}$ that this CME was interacting with the southern streamer at the angle PA2. We consider that the part of CME away from its nose and interacting with the nearby streamer should correspond to the CME flank. This is why we believe that PA2 should correspond to the southern flank of the CME. Since there was a data gap between 17:32 UT and 17:44 UT in MLSO/MK4 observation, the CME was first seen only at 17:44 UT in the MK4 field of view.

\subsection{Radio observations}

The GBSRBS is located in a radio quiet zone at NRAO's Green Bank site, and produces high quality radio dynamic spectra with low noise interferences in the range from $18 \mathrm{MHz}$ to $70 \mathrm{MHz}$ with 565 frequency channels with $1 \mathrm{~s}$ time resolution (White et al. 2005). The low noise is an advantage for our purpose of estimating the type II burst frequencies. We use the background subtracted dynamic spectrum, which is available at the website ${ }^{2}$ of Green Bank observatory.

Figure 2 shows the GBSRBS dynamic spectrum together with lightcurves of GOES 0.5-4 $\AA$ flux (dashed line) and total radio flux integrated from $18 \mathrm{MHz}$ to $70 \mathrm{MHz}$ (solid line). The type II burst started at 17:44 UT, just at the first appearance time of the CME in the MK4 coronameter. While the harmonic band of the type II burst started at 17:46 UT and lasted for about $40 \mathrm{~min}$ from above $70 \mathrm{MHz}$ to $20 \mathrm{MHz}$, the fundamental band has a frequency drifting from about $60 \mathrm{MHz}$ at 17:44 UT to $18 \mathrm{MHz}$ at $17: 58 \mathrm{UT}$.

\section{Analysis}

\subsection{Onset time}

The CME first appears on the MLSO images at 17:44 UT. The onset time of the CME (i.e. the onset time of the eruption leading to the CME) is estimated to be $\sim 17: 32$ UT if we extrapolate the height-time plot to the solar surface using the height $2.06 R_{\odot}$ and the speed $878 \mathrm{~km} \mathrm{~s}^{-1}$ of the CME at 17:46 UT (see Table 1). This well agrees to the onset time of the eruptive prominence (17:32-17:34 UT) as we found in BBSO $\mathrm{H} \alpha$ and TRACE EUV

\footnotetext{
${ }^{2}$ http://www.nrao.edu/astrores/gbsrbs/
} 
Table 1. Kinematics of CME and type II shock.

\begin{tabular}{|c|c|c|c|c|c|c|c|c|c|}
\hline \multirow[b]{2}{*}{$\begin{array}{l}\text { Time } \\
\text { (UT) }\end{array}$} & \multicolumn{3}{|c|}{ CME front } & \multirow[b]{2}{*}{$M_{\mathrm{A}}$} & \multicolumn{3}{|c|}{ CME flank } & \multirow[b]{2}{*}{$M_{\mathrm{A}}$} & \multirow{2}{*}{$\begin{array}{c}\text { Type II } \\
V_{\text {II }} \\
\left(\mathrm{km} \mathrm{s}^{-1}\right)\end{array}$} \\
\hline & $\begin{array}{c}\text { Height } \\
\left(R_{\odot}\right)\end{array}$ & $\begin{array}{c}V_{\mathrm{CME}} \\
\left(\mathrm{km} \mathrm{s}^{-1}\right)\end{array}$ & $\begin{array}{c}V_{\mathrm{A}} \\
\left(\mathrm{km} \mathrm{s}^{-1}\right)\end{array}$ & & $\begin{array}{c}\text { Height } \\
\left(R_{\odot}\right)\end{array}$ & $\begin{array}{c}V_{\mathrm{CME}} \\
\left(\mathrm{km} \mathrm{s}^{-1}\right)\end{array}$ & $\begin{array}{c}V_{\mathrm{A}} \\
\left(\mathrm{km} \mathrm{s}^{-1}\right)\end{array}$ & & \\
\hline $17: 46$ & 2.06 & 878 & 526 & 1.6 & 1.72 & 786 & 392 & 2.0 & 700 \\
\hline $17: 49$ & 2.25 & 583 & 630 & 0.9 & 1.91 & 751 & 358 & 2.1 & 655 \\
\hline $17: 52$ & 2.41 & 747 & 692 & 1.1 & 2.09 & 632 & 365 & 1.7 & 600 \\
\hline mean & & 736 & 616 & 1.2 & & 723 & 372 & 1.9 & 652 \\
\hline
\end{tabular}

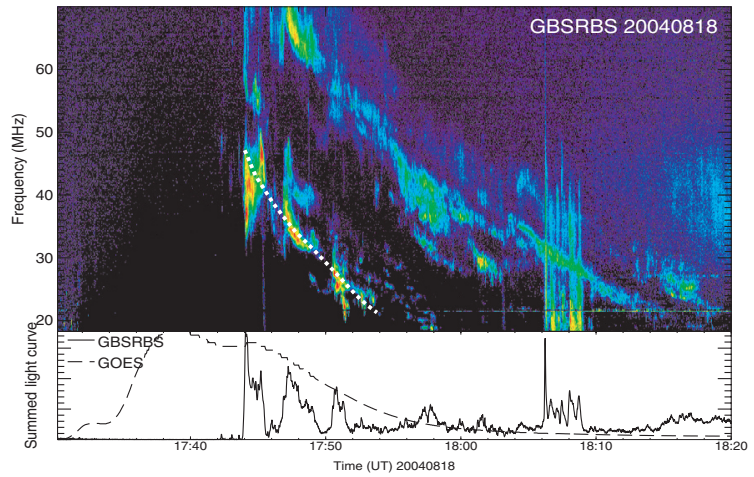

Fig. 2. Dynamic spectrum of the type II burst observed by the GBSRBS on 2004 August 18. Dotted white line in the upper panel indicates the frequency drift of the fundamental emission band of the burst. The dashed and solid lines in lower panel denote the GOES X-ray flux and total radio flux curve, respectively.

(1600 ̊) images. On the other hand, the starting time of type II burst rather coincides with the first appearance of the CME in MK4 field of view.

\subsection{Coronal density}

We derive the coronal density distribution by inversion of polarized brightness measurement (van de Hulst 1950) using the LASCO IDL routine (pb_inverter.pro) in the SolarSoft package $^{3}$. Detailed description of the routine can be found in Hayes et al. (2001). The left panel of Fig. 3 shows the selected MLSO coronal polarization brightness image. This data was taken at 17:06 UT, before the CME onset time (17:32-17:34 UT), and thus represents the background coronal density. The lines denoted with a to $\mathrm{h}$ are the selected position angles ranging from $219^{\circ}$ to $288^{\circ}$ that encompass the entire area later swept by the CME. Shown in the right panel is a difference image between two MLSO images at 17:06 UT and 17:03 UT. Since the difference image exhibits no noticeable plasma motion, we assume that our density measurement will not be contaminated by any mass motion.

Figure 4 shows the measured density distributions along the selected position angles (defined in Fig. 3) by using an averaged polarization data taken at 17:06 UT, which is usually acquired once a day from 5 sequential images. As comparison, we also plot density distributions from a popular model, the one and two-fold Newkirk models (Newkirk 1961) denoted as NK1 and NK2, respectively. As shown in the figure, the derived electron densities near southern helmet streamer $(b, c, d)$ are most enhanced, lying between one-fold (NK1) and two-fold (NK2) Newkirk models at some heights $\left(1.6-2.2 R_{\odot}\right)$. The density found along the position angle (e), that is swept by CME nose is less than NK1 at height above $1.8 R_{\odot}$. The northern streamer

\footnotetext{
${ }^{3}$ http://www. lmsal.com/solarsoft
}



Fig. 3. MLSO coronal polarization brightness data (left) just before the CME event and its difference image (right). Coronal density was measured along the dotted lines at 9 position angles $\left(a=219^{\circ}, b=225^{\circ}\right.$, $\mathrm{c}=231^{\circ}(\mathrm{PA} 2), \mathrm{d}=237^{\circ}, \mathrm{e}=250^{\circ}(\mathrm{PA} 1), \mathrm{f}=270^{\circ}, \mathrm{g}=276^{\circ}, \mathrm{g}^{\prime}=282^{\circ}$, and $\mathrm{h}=288^{\circ}$, respectively).



Fig. 4. Measured coronal density distributions along the position angles denoted in Fig. 3. The distributions at the northern flank of the CME at $276^{\circ}(\mathrm{g})$ and $282^{\circ}\left(\mathrm{g}^{\prime}\right)$ are similar to each other. Dotted lines denoted as NK1 and NK2 represent the one- and two-fold Newkirk density models, respectively.

region ( $\mathrm{f}, \mathrm{g}, \mathrm{h}$ ) shows the least amount of density in the high corona.

We note that another small CME had occurred before the CME under current investigation. According to LASCO data it first appeared at 17:30 UT at a height of $2.36 R_{\odot}$ near the southern streamer, and later ( $>18: 05$ UT) was cannibalized by our CME, similar to the CME interaction case described by Gopalswamy et al. (2001b, 2004). Depending on how significantly the preceding CME had modified the background density at 17:06 UT, the density derived above may or may not correctly represent the background density at the type II shock forming time (17:44 UT). We thus carefully re-examined all MLSO MK4 difference images between 17:06 and 17:32 UT to find any CME signature near the active region (S14W90). However, we detected no obvious feature of the preceding CME in all difference images. Perhaps the intensity of the preceding CME was 


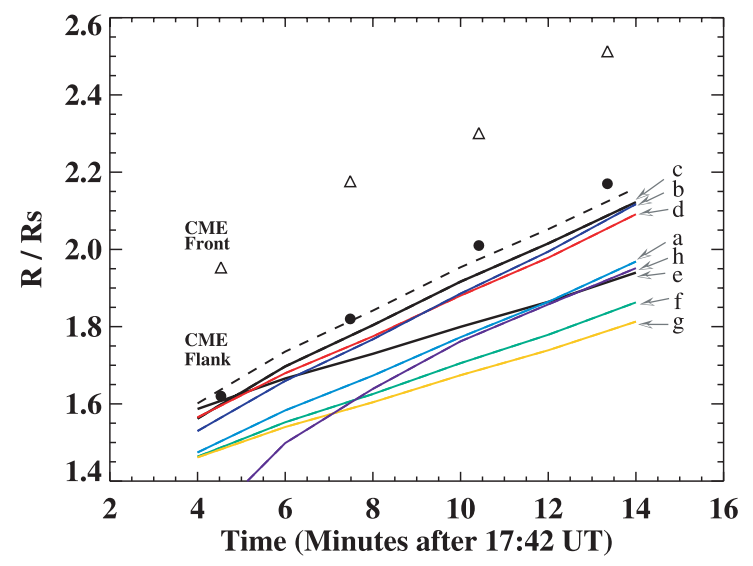

Fig. 5. Height-time plot of CME front (triangles) and CME flank (filled circles). Each line denotes the coronal shock height estimated from the radio data and the measured coronal density distribution along the position angles. Dotted line represents the shock height estimated by assuming $20 \%$ density enhancement along PA2 during the period between 17:15 and 17:32 UT.

too low to be detected by the MLSO MK4 coronameter. Note that MK4 is considerably less sensitive to CMEs than LASCO because MK4 measures polarization brightness of photosphere radiation scattered by free electrons in the corona, while LASCO measures coronal radiation in total intensity. While the CME itself was not detected in MLSO images, the density variation caused by that CME could have affected the MLSO images. We thus determine the density distribution at the CME-streamer interface (PA2 in Fig. 3) by using all available images between 17:06 and 17:32 UT and then compare to the density profile that is estimated by using the averaged polarization data taken at 17:06 UT. We found about $20 \%$ density enhancement after 17:15 UT in the range between 1.4 and $2.2 R_{\odot}$ due to the preceding CME. This time-dependent density is taken into account in the next section.

\subsection{Locations of the type II burst and CME}

We use the measured density distributions (Fig. 4) in converting the observed type II frequencies to the shock heights. We first measure the emission frequencies in the fundamental band (the white dotted line in dynamic spectrum of Fig. 2), and estimate the corresponding electron densities under the assumption that the radio emission is generated at plasma frequency $\left(f_{\mathrm{p}}=9000 \sqrt{N_{\mathrm{e}}}[\mathrm{Hz}]\right)$ due to the disturbed electrons by the type II shock. The emission heights are deduced from the density function determined from a polynomial fitting of the measured density profiles (Fig. 4). We measure the CME heights using the vignetted white-light images from MLSO, at its nose and flank (i.e., along the dotted lines in Fig. 1 from 17:44 UT to $17: 53 \mathrm{UT})$. The measurement error for the CME height, as deduced from 10 trial measurements of a CME position in the MK4 vignetted image, is about $0.01 R_{\odot}$ (about 2-pixel) and that of speed is about $95 \mathrm{~km} \mathrm{~s}^{-1}$, respectively.

Figure 5 shows the type II shock heights calculated along the selected position angles (solid lines) in comparison with the CME heights (symbols). Triangles and filled circles correspond to the $\mathrm{CME}$ front and the $\mathrm{CME}$ flank, respectively. The lines e and c correspond to the shock heights estimated along the CME front (PA1 in Fig. 3) and the southern helmet streamer (PA2 in Fig. 3), respectively. For the first $\sim 10 \mathrm{~min}$, the CME front propagated from 1.9 to $2.5 R_{\odot}$ and the flank followed it by a nearly constant distance (about $0.3 R_{\odot}$ ). It appears

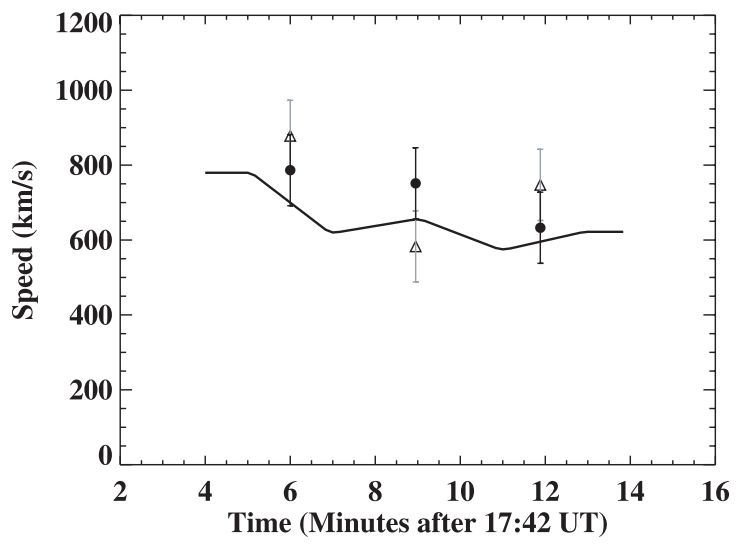

Fig. 6. Speed comparison among coronal shock (solid line) estimated density profile (c) along southern CME flank, CME front (triangles), southern CME flank (filled circles). The error bars indicates the uncertainty $\left( \pm 95 \mathrm{~km} \mathrm{~s}^{-1}\right)$ of CME speed due to measurement errors (2-pixels) in MK 4 image.

that the type II shock heights, estimated from the density profile measured near the southern helmet streamer (c, b, d) contacting with the CME, are consistent with the heights of the southern CME flank (filled circles). All the others show a large deviation from both CME front (triangles) and CME flank (filled circles). We thus regard that type II burst was generated at the place where the CME interacts with the streamer at the southern flank.

The dotted line in Fig. 5 represents the shock height when we take into account the time dependent density enhancement. In this case the type II shock heights better agree with those of the southern CME flank. This suggests that there could indeed be additional density enhancement ( 20\%) along the CME flank due to the passage of the preceding CME along the streamer.

\subsection{Speeds of the type II burst and CME}

As we mentioned in Sect. 1, imaging comparison is the best way to inspect the relationship between type II bursts and CMEs, but in its absence, one has to use density measurements. In Fig. 6 we compare the speed of the type II burst with that of the CME. Since the heights of the type II source and CME flank coincide, their speeds also agree to each other within the error range. Note however that the CME front also has similar speed, because the CME flank followed the front by a nearly constant distance behind. It is thus our view, based on this single event, that the speed and timing alone cannot tell at which part of the CME the type II emission is generated, and that locating ability (such as in Fig. 5) is necessary to address the origin of type II bursts.

Another noteworthy thing in this figure is that the type II speed tends to be nearly constant (or slowly decreasing) with time. This behavior is inconsistent with that of the flare waves associated with type II bursts, e.g., Moreton waves. Usually the Moreton waves show a strong deceleration at about $-1500 \mathrm{~m} \mathrm{~s}^{-2}$, which is thought as they are generated by initially strong shocks by flares (e.g., blast waves) and then decay to fast mode waves with strong deceleration (Warmuth et al. 2004a,b). The nearly constant shock speed shown in Fig. 6 is rather consistent with the behavior of a piston driven shock by a CME (Leblanc et al. 2001).

Table 1 summarizes the kinematics of the CME and type II shock. As mentioned, the speeds of the CME flank and type II shock are comparable to each other within the MK4 CME measurement error $\left( \pm 95 \mathrm{~km} \mathrm{~s}^{-1}\right)$. A mean speed of type II shock is about $650 \mathrm{~km} \mathrm{~s}^{-1}$ that is similar to those of CME flank 
$\left(720 \mathrm{~km} \mathrm{~s}^{-1}\right)$ and CME front $\left(740 \mathrm{~km} \mathrm{~s}^{-1}\right)$. Both the type II shock speed and the CME flank speed show a similar tendency of weak deceleration. The local Alfvén speeds are calculated by adopting the active region magnetic field model (Dulk \& Mclean 1978), which was first used by the Gopalswamy et al. (2001a) to estimate Alfvén speed profile, together with the measured MK4 density distributions along the CME front and the CME flank, respectively (Fig. 4). It appears that the Alfvénic Mach number is 1.2 at the CME front and 2.0 at the CME flank, suggesting that the latter is more favorable for generating the shock.

\section{Conclusion}

In this paper, we analyzed an event of metric type II burst and a CME that occurred on 2004 August 18 to understand their relationship. We excluded from the beginning the possibility of flare produced type II shock because of the inconsistent timing between the type II burst and the flare impulsive phase. It is later found through the analysis that the shock speed is nearly constant, which is also against the behavior of shock speed found for the flare associated events (Warmuth et al. 2004a,b).

Our analysis was focused on not only whether the type II bursts is related to the CME, but at which part of the CME the type II burst was generated. Our technique used for this purpose is to compare, for agreement, the densities inferred from the GBSRBS type II spectrum with those calculated from the two-dimensional polarization map of MLSO/MK4 coronameter. It turns out that this technique based on 2D density measurement is sensitive enough to locate the type II emission source within the width of the $\mathrm{CME} \sim 30^{\circ}$. Of course, this locating capability of our technique is not only limited by the pixel resolution of the MLSO coronameter, but depends on the density inhomogeneity in the region of interest. If the electrons were distributed in the corona with real radial symmetry, our technique would make little difference from other works based on one-dimensional density models. In this event, the presence of a streamer caused a nonradial density contrast by a sufficient amount, and we were able to distinguish whether the type II burst is generated at the CME nose or at its flank $\left(\sim 20^{\circ}\right.$ away from the nose $)$.

Our result that the type II burst was generated along the flank of the CME interacting with the adjacent streamer agrees to the earlier speculations presented by Classen \& Aurass (2002), Reiner et al. (2003), Mancuso \& Raymond (2004), and Cho et al. (2005). A difference between the latter studies and ours is that we here attempt to locate the shock within a partial area of the $\mathrm{CME}$, whereas in other studies the idea was inferred from inconsistent locations of the CME front and the type II source based on a one-dimensional density model. Not only the location but also the nearly constant speed of the type II emission source found in this study supports the existing idea that the type II shock could be bow/piston driven by the top or the flanks of CMEs when they interact with the high density structure of the corona.

We also found that the electron density along the southern streamer was already more enhanced than other neighboring regions before being swept by the CME. To be more specific, the density distribution along the streamer is comparable to that of the one-fold Newkirk model in the type II formation regions $\left(1.6 R_{\odot}\right.$ and $\left.2.3 R_{\odot}\right)$, whereas at the other region including northern streamer the density falls below the Newkirk model. Such density contrast can lead to different Alfvénic Mach numbers along the CME, although the speed of the CME flank is comparable to that of the CME front $\left(800\right.$ to $\left.600 \mathrm{~km} \mathrm{~s}^{-1}\right)$. This CME speed is consistent with the average speed of metric type II associated CMEs (Gopalswamy et al. 2005).
We used an active region magnetic field model (Dulk \& McClean 1978) to estimate the Alfvénic Mach number of $\sim 1.2$ at the CME nose and $\sim 2$ in the CME flank. This may account for our result in that the CME flank's mass motion moves with a speed that exceeds the local Alfvénic values and, thus, is favorable for generating the type II shocks.

While the present result for a single event strongly supports the scenario of the type II shock generation at the CME flank interacting with the nearby streamer, a more extended study will be required in order to draw a more definite conclusion on the association between type II and CME.

Acknowledgements. The authors are very grateful to N. Gopalswamy for his valuable comments and suggestions that help to improve the manuscript. We thank Joan T. Burkepile and S. White for providing the MLSO/MK4 data and GBSRBS data, respectively. K.S.C. gratefully acknowledges the help of R. A. Howard in estimation of coronal density using MLSO pB data. This work has been supported by the MOST funds (M1-0104-00-0059 and M1-040700-0001) of the Korean Government. J.L. has been supported by NSF under grant AST-0307670 to New Jersey Institute of Technology. Y.J.M. and Y.D.P. were supported by the Korea Research Foundation (KRF-2005-070-C00059) of the Korean Government. M.D. appreciates the hospitality of the NOAA Space Environment Center during his emeritus tenure.

\section{References}

Bemporad, A., Poletto, G., Suess, S. T., et al. 2003, ApJ, 593, 1146

Cho, K.-S., Moon, Y.-J., Dryer, M., et al. 2003, J. Geophys. Res., 108(A12), SSH 8-1

Cho, K.-S., Moon, Y.-J., Dryer, M., et al. 2005, J. Geophys. Res., 110(A12), A 12101

Classen, A., \& Aurass, H. 2002, A\&A, 384, 1098

Cliver, E. W., Webb, D. F., \& Howard, R. A. 1999, Sol. Phys., 187, 89

Dulk, G. A., \& McLean, D. J. 1978, Sol. Phys., 57, 279

Elmore, D. F., Burkepile, J. T., Darnell, J. A., Lecinski, A. R., \& Stanger, A. L. 2003, Proc. SPIE, 4843

Gary, D. E., Dulk, G. A., House, L., et al. 1984, A\&A, 134, 222

Gopalswamy, N., Kundu, M. R., Manoharan, P. K., et al. 1997, ApJ, 486, 1036

Gopalswamy, N., Kaiser, M. L., Lepping, R. P., et al. 1998, J. Geophys. Res., 103,307

Gopalswamy, N., Lara, A., Kaiser, M. L., \& Bougeret, J.-L. 2001a, J. Geophys. Res., 106, 25261

Gopalswamy, N., Yashiro, S., Kaiser, M. L., Howard, R. A., \& Bougeret, J.-L. 2001b, ApJ, 548, L91

Gopalswamy, N., Yashiro, S., Krucker, S., Stenborg, G., \& Howard, R. A. 2004, J. Geophys. Res., 109, A12105

Gopalswamy, N., Aguilar-Rodriguez, E., Yashiro, S., et al. 2005, J. Geophys. Res., 110, A12

Guhathakurta, M., \& Fisher, R. 1998, ApJ, 499, L215

Hayes, A. P., Vourlidas, A., \& Howard, R. A. 2001, ApJ, 548, 1081

Lara, A., Gopalswamy, N., Nunes, S., Munoz, G., \& Yashiro, S. 2003, Geophys. Res. Lett., 30(12), 8016

Leblanc, Y., Dulk, G. A., Vourlidas, A., \& Bougeret, J.-L. 2001, J. Geophys. Res., 160, 25, 301

Maia, D., Pick, M., Vourlidas, A., \& Howard, R. 2000, ApJ, 528, L49

Mancuso, S., \& Raymond, J. C. 2004, A\&A, 413, 363

Mancuso, S., Raymond, J. C., Kohl, J., Ko, Y.-K., \& Wu, R. 2003, A\&A, 400, 347

Newkirk, G. Jr. 1961, ApJ, 133, 983

Parenti, S., Bromage, B. J. I., Poletto, G., et al. 2000, A\&A, 363, 800

Reiner, M. J., Vourlidas, A., Cyr, O. C. St., et al. 2003, ApJ, 590, 533

Robinson, R. D., \& Stewart, R. T. 1985, Sol. Phys., 97, 145

Shanmugaraju, A., Moon, Y.-J., Dryer, M., \& Umapathy, S. 2003, Sol. Phys., 215,164

Thompson, R. J., Kennewell, J. A., \& Prestage, N. P. 1995, Sol. Phys., 166, 371. van de Hulst, H. C. 1950, Bull. Astron. Inst. Netherlands, 11 (410), 135

Warmuth, A., Vrsnak, B., Magdalenic, J. Hanslmeier, A., \& Otruba, W. 2004a, A\&A, 418, 1101

Warmuth, A., Vrsnak, B., Magdalenic, J., Hanslmeier, A., \& Otruba, W. 2004b, A\&A, 418, 1117

White, S. M., Bastian, T. S., Bradley, R., Parashare, C., \& Wye, L. 2005, in From Clark Lake to the Long Wavelength Array: Bill Erickson's Radio Science, ed. N. E. Kassim, M. R. Perez, W. Junor, \& P. A. Henning, ASP Conf. Ser., 345,176

Wild, J. P., \& McCready, L. L. 1950, Austral. J. Sci. Res., A3, 387 